\title{
PERICARDITIS WITH EFFUSION. AN EXPERIMENTAL STUDY*
}

CHARLES SPENCER WILLIAMSON, M.D.

There can scarcely be any better comment on the difficulty in diagnosticating pericarditis with effusion than can be read out of statistics from the postmortem room. Even in institutions where the diagnostic work is admittedly of a high order, the postmortem findings of pericardial exudates entirely unsuspected during life is of not infrequent occurrence. Any one who has been especially interested in the subject must have been struck with the difficulties which are at times encountered in making the diagnosis of such an effusion. Of course, when a well defined friction rub develops in the course of rheumatism, and is followed by a gradual increase in the cardiac silhouette, there can be little difficulty in the diagnosis. On the other hand, in the absence of a pericardial rub, the differentiation between a dilated heart and an effusion may really be very difficult. A considerable number of cases have been reported in which the pericardial sac was aspirated with the expectation of finding fluid, but instead of fluid pure blood came through the needle, in such quantities as to indicate that the heart wall had been punctured. On several occasions I have seen men of distinguished ability do a paracentesis cordis instead of a paracentesis pericardii.

When we examine into the current descriptions of the physical signs in pericarditis, and particularly when we investigate to see on what researches these are based, we meet with a confusion of ideas and statements. While not wishing to discuss the subject from a historical standpoint, a brief statement and criticism of the most important views would seem in place.

One of the oldest views, championed especially by Skoda ${ }^{1}$ and his followers, ${ }^{2}$ treats of the relations of the heart to the pericardium very

* From the departments of medicine and anatomy, College of Medicine, University of Illinois.

* Although the casts forming the basis of this paper were exhibited at the 1917 meeting of the American Medical Association, my absence in military service has precluded the earlier publication of the paper.

1. Skoda: Abhandl. ueber Auscultation und Percussion, Wien., 1839.

2. Oppolzer: Vorlesungen ueber die Krankheiten des Herzens, 1867.

Nore.-In each case the left hand photograph is a picture of the cast of the heart itself; the middle photograph is a cast of the corresponding exudate, and the photograph on the right is a section through the exudate on the dotted line. The chest outline shows the relations of the heart and the exudate as seen from the front. 

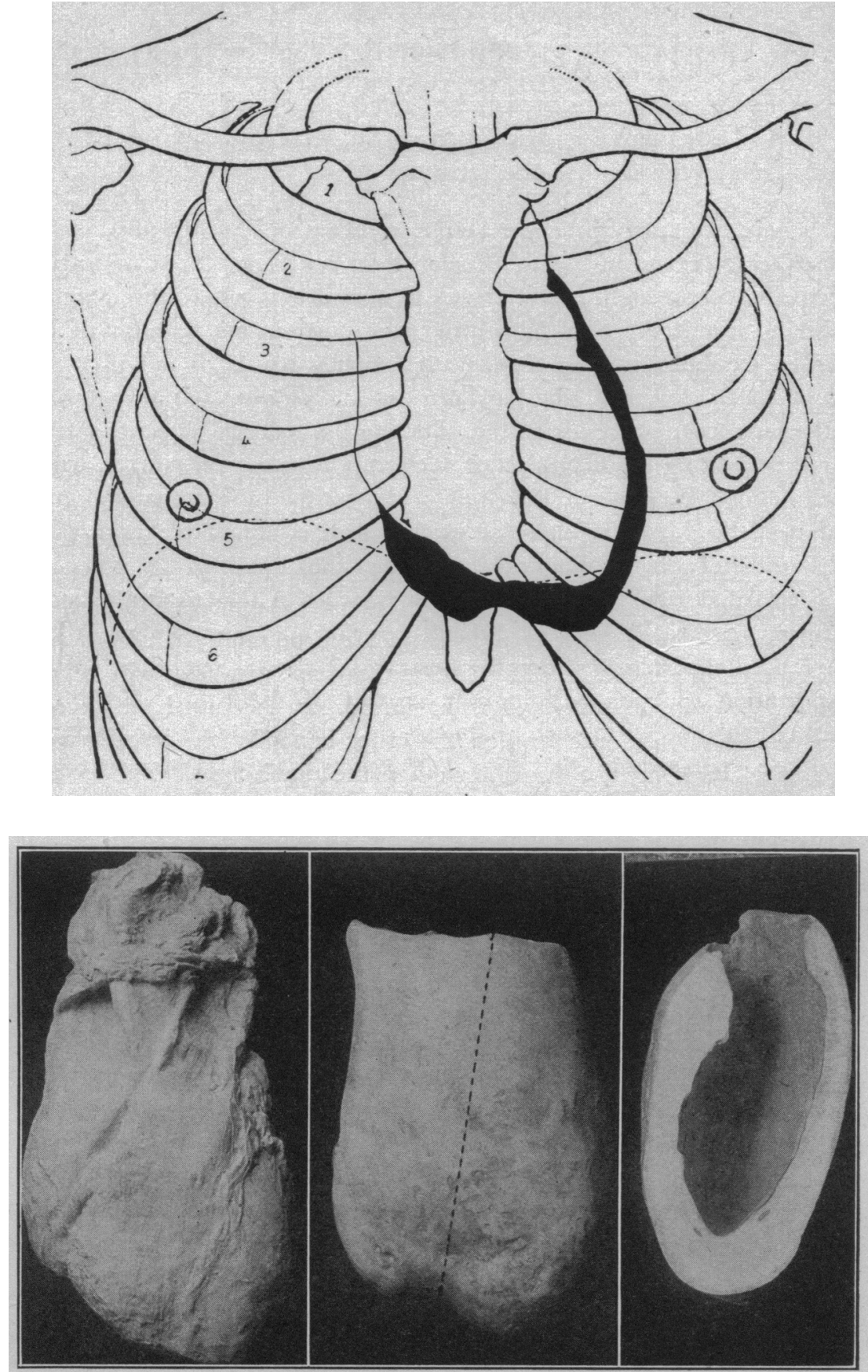

Fig. 1. - Exp. 4; 300 c.c. injected. In addition to the accumulation of fluid on the diaphragmatic surface and around the apex, there is a considerable amount in front of the great vessels at the base. (See note, page 206.) 
much in the manner of a vessel of water containing a solid body, the specific gravity of which is greater than that of the water. Inasmuch as the heart, with its contents, has a higher specific gravity than a pericardial effusion, it is manifest that on this theory the heart must needs sink in the fluid to the lowest point of the pericardial sac. It requires, however, only a superficial examination to see that the situation is by no means so simple as this view would lead us to believe. To begin with, the analogy of a solid body sinking in a vessel full of water is far from a correct one, especially with small amounts of fluid. The pericardium is not a vessel with rigid walls, but a tough membrane lying with a large part of its surface in close contact with the heart. It is reflected onto the great vessels at the base, and intimately united with the diaphragm at the central tendon of the latter. It will hold about 100 c.c. of fluid before it begins to be stretched. To surround the heart entirely with a layer of fluid of sufficient depth so that one can talk of the heart "sinking" or "swimming" requires an exudate of considerable size, as can readily be seen from our casts. A second and even more important reason why the heart cannot "sink" freely in the fluid, even when this is present in large amounts, is seen in the attachments of the heart. This fixation, as has been shown by several authors, is quite definite and is produced, on the one hand, by the great vessels at the base, and on the other hand, by the inferior vena cava which anchors the heart firmly to the diaphragm. The axis of fixation extends from the inferior vena cava below, to a point a little to the left of the median line above, which may be regarded as the point of application of the traction of the great vessels. It is plain, therefore, that the only extensive movement of the heart which can occur in pericardial effusion, is of the apical portion.

Quite the opposite of this view is held by Schaposchnikoff ${ }^{3}$ and his followers. After a series of experiments, using fluid injections, this author came to the conclusion that the heart swims in the fluid and believes that it is held in this position by the elastic traction of the great vessels. This view is strongly supported, he thinks, by the fact that even with large exudates a loud friction rub may be heard over the entire sternum, and at necropsy the heart may be found in close contact with the sternum. Furthermore, numerous operations on purulent effusions have shown that when an incision is made through the chest wall, the heart itself presents in the wound, and that the purulent exudate can only be reached by pushing the heart out of the way. A case of this sort was reported by $\mathrm{me}^{4}$ and the patient was operated

3. Schaposchnikoff, B.: Mitt. a. d. Grenzgel,. d. Med. u. Chir. 2:86, 1897. Rev. de Méd. 2:789, 1905.

4. Med. Clin. 2:907 (March) 1917. 

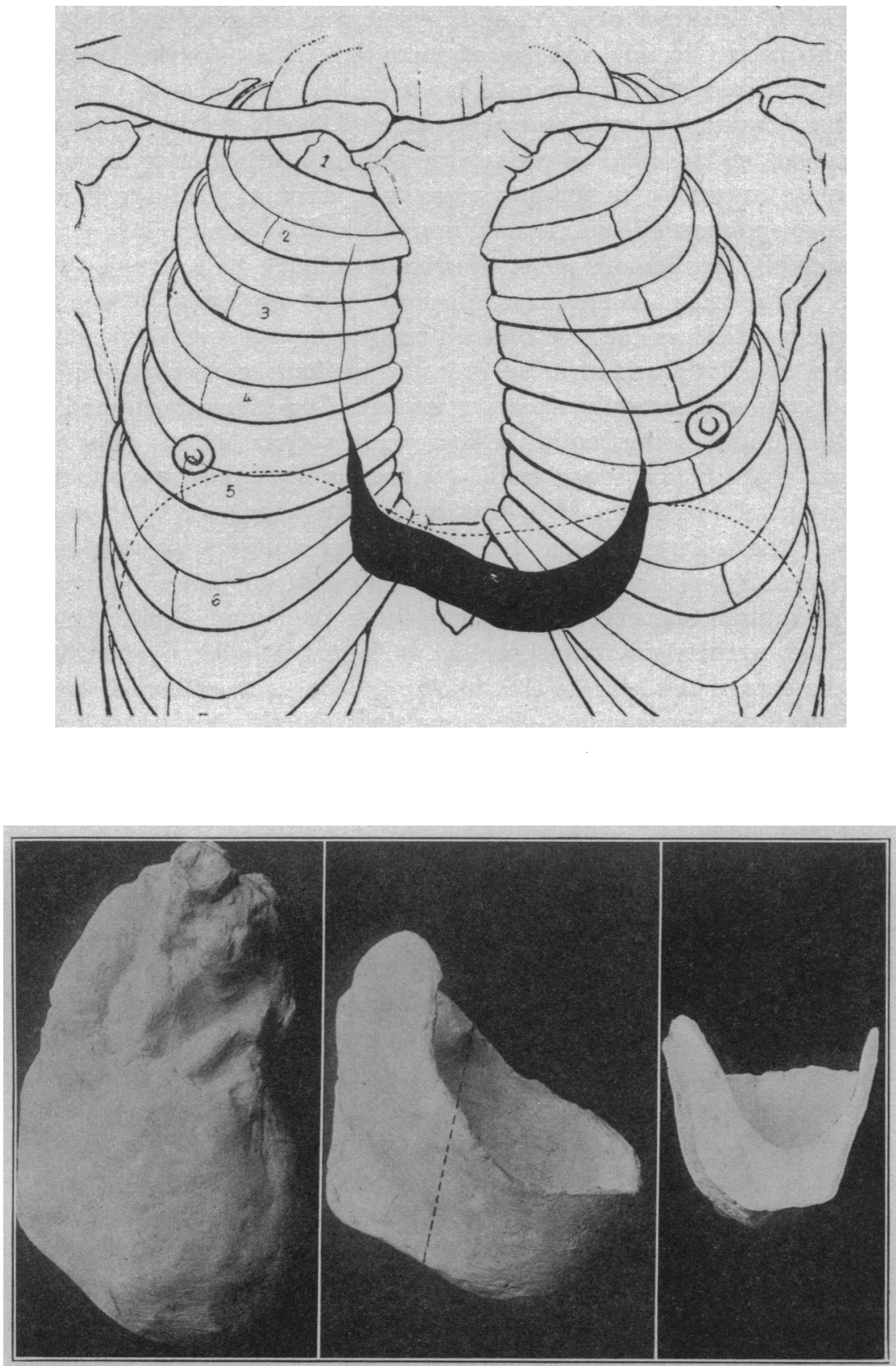

Fig. 2.-Exp. 18; 240 c.c. injected. A typical small sized exudate is present, confined almost entirely to the diaphragmatic surface. (See note on page 206.) 
on by Dr. Kellogg Speed. The heart was in close contact with the chest wall, and no pus could be obtained until it was pushed back out of the way and the drainage tube inserted.

Curschmann, ${ }^{5}$ writing still more recently, holds a view at variance with those of the other observers mentioned. This author holds that the more important factor governing the distribution of the fluid, is the small amount of space which normally exists between heart and sternum in front and the vertebral column behind. He holds that there is very little room for the heart either to "sink" or "swim." The fluid poured out follows the line of least resistance, and not being able to expand anteriorly and posteriorly in the median line, must, of necessity, spread out laterally toward the pleural cavities, or upward and downward in the direction of the great vessels above and the diaphragm below. This author supports his contention by a few cross-sections.

Rotch, of Boston, in 1878 investigated the behavior of the fluid by injecting cacao butter into cadavers and determining by percussion the point of first appearance of the fluid. The subjects had been previously tracheotomized and the lungs inflated until they corresponded in size with the outlines given by Luschka. The most serious objection to Rotch's results is found in that of the sixteen cadavers used-twelve were those of young infants - and that the method is highly subjective. Rotch's results, however, have been quoted widely, and his main contention that a dullness in the fifth intercostal space on the right side is the first sign of a pericardial exudate, has been accepted by many writers. ${ }^{6}$ Ebstein $^{\top}$ in particular, whose attention was called to this sign by reading Rotch's article, ${ }^{8}$ expresses essentially the same thought in another way. The angle between the right heart and the liver, the so-called cardiohepatic angle, is normally acute, and he believes that the rounding of this angle is the first clinical expression of a pericardial exudate, and supports this by many illustrative clinical cases. Inasmuch as these findings were all obtained by percussion only, his results lie open to the criticism of subjectivity. Many experienced clinicians have failed to find either the dullness in the fifth right interspace or the rounding of the cardiohepatic angle in proven cases of pericardial effusion.

It is evident that all of these theories cannot be, without modification, reconciled with each other, and with the idea of attempting to bring some order out of the chaos, the experimental work in this research was undertaken.

5. Curschmann, H.: Die Deutsche Klinik am Eingang des Zwanzigsten Jahrhunderts 4:401, 1905 .

6. Aporti u. Figaroli: Zentralbl. f. inn. Med. 21:737, 1900.

7. Ebstein: Ueber die Diagnose beginnender Fluessigkeitsansammlungen im Herzbeutel, Virchows Arch. f. path. Anat. 130:418.

8. Rotch: Boston M. \& S. J. Sept. 26, 1878. 

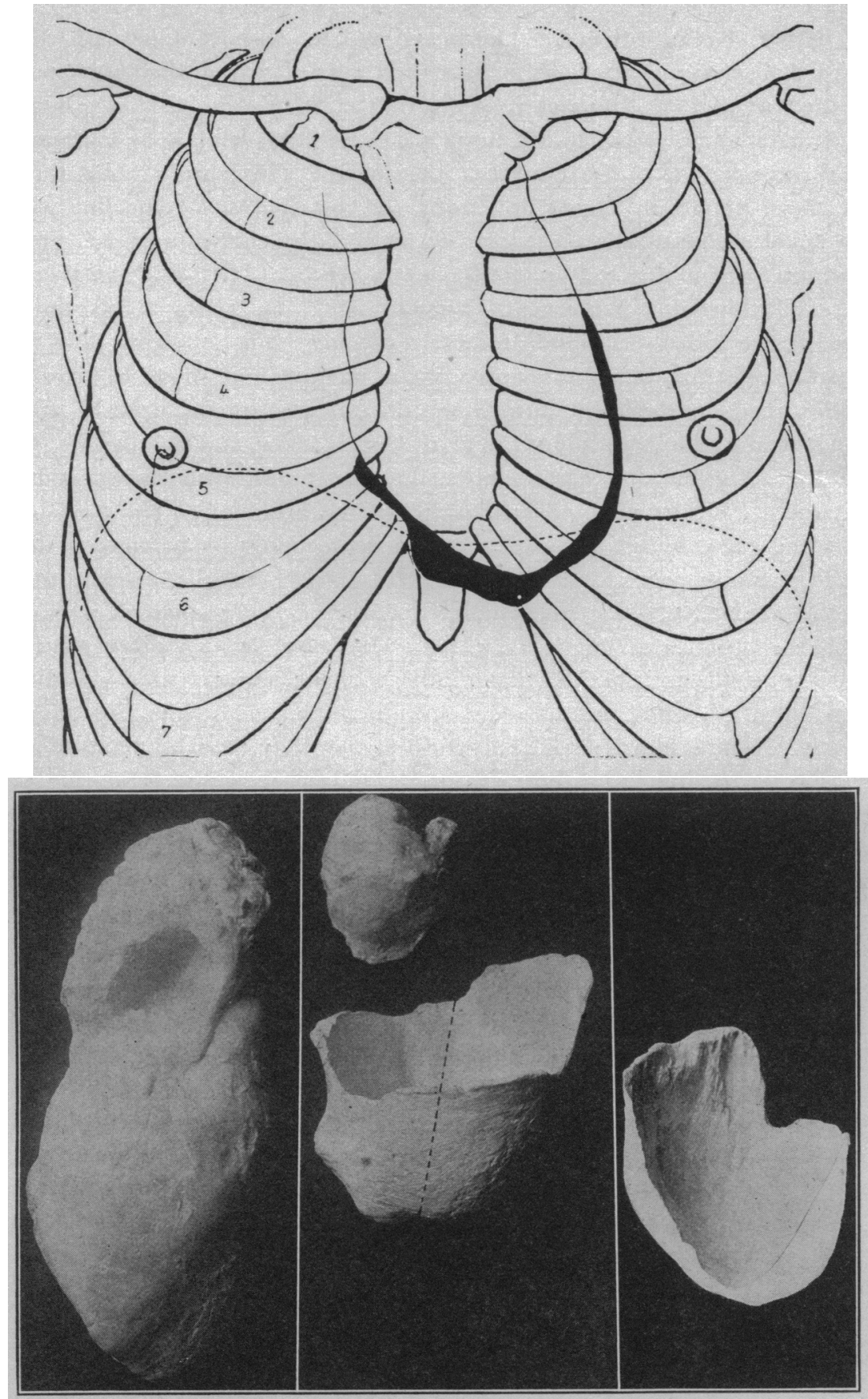

Fig. 3.-Exp. 26; 175 c.c. injected. The fluid is almost entirely limited to the diaphragmatic surface and covering the border of the right ventricle. (See note on page 206.) 


\section{EXPERIMENTAL WORK}

Methods Employed.-The most important point in any method is that it shall possess a high degree of objectivity. All methods which depend on clinical observations during life lack this almost entirely, and very little progress has been made with these. Much was expected from the applications of the roentgen ray, but however great its usefulness as a diagnostic accessory, it is of relatively small scientific value in this connection, in that it is open to grave errors of interpretation. When used in the ordinary way, namely, with the plate either on the front or back of the chest, it fails entirely to show fluid accumulations either in front or behind the heart.

The most satisfactory methods for experimentation are those which depend on injecting fluids into fresh cadavers. A number of authors have done this after removal of the sternum, which, I believe, entirely invalidates the work, in that it permits the fluid to accumulate in front of the heart by removing the principal obstacle to its accumulation at that point. A far better plan is to make the injection through the central tendon of the diaphragm, since this can be done without materially disturbing the abdominal relations. I selected for the position of the cadaver that most generally used in clinical examination, such as would be represented by the patient being propped on a pillow at an angle of about 15 degrees. Some of our injections were made with the body at an angle of 120 degrees with the thighs, i. e., in the orthopnea position.

After a great deal of preliminary experimentation, I settled on the following technic, which after the requisite manual dexterity had been acquired, proved to be very satisfactory. A small incision was. made in the costoxiphoid angle, through which, guided by a finger, a long specially designed trocar was passed. This was made of tempered steel with a sharp stilet provided with a ball on the outside, from threeeighths to one half inch from the point. The purpose of this ball was to prevent penetration to too great a distance. To make the injection, the trocar was thrust obliquely through the central tendon of the diaphragm, the stilet was then withdrawn and a large brass syringe attached to the needle by a screw thread. The syringe I used held 300 c.c. and was provided with a screw arrangement by which the plunger could be depressed slowly and evenly.

As an injection fluid I settled on a mixture of gelatin and agaragar made up to the proper specific gravity by the addition of salt. Such a solution, when warm, is perfectly fluid, and under our conditions of experiment, required from one-half hour to one hour to harden sufficiently so as not to be influenced by change of position. To make sure that coagulation would be thorough, we allowed from 

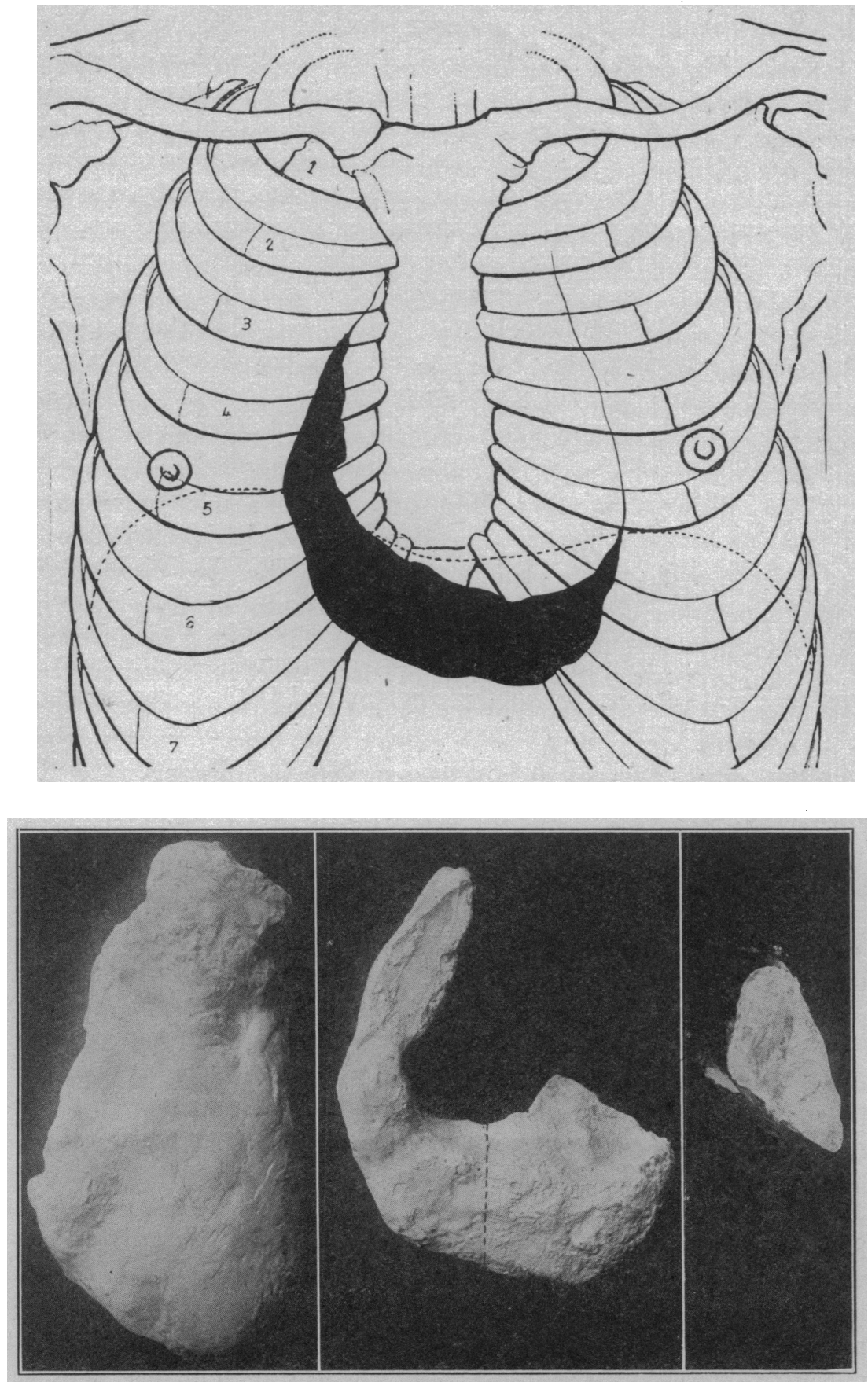

Fig. 4.-Exp. $29 ; 225$ c.c. injected. The larger portion of fluid is on the diaphragmatic surface, pushing down the liver markedly. A thin tongue of fluid runs along the right margin of the heart. (See note on page 206.) 
twelve to twenty-four hours to elapse before making the necropsy. All the information which could be elicited by percussion was obtained as the injections progressed. In each case accurate measurements were taken of the thorax circumference and of the distance between the posterior surface of the sternum and the anterior surface of the vertebral column. At the necropsy the fenestrum was carefully removed, and the exact positions of the heart and exudate were measured, the general outline of the heart and pericardium studied, and then the entire pericardial sac and great vessels were removed in toto. The exudate was sufficiently hard so that it could be handled without fear of breaking. It was immediately taken to the laboratory, placed in a low temperature icebox, and thoroughly frozen. The hearts were then turned over to Mr. Hammer, the modeler of the University of Illinois, whose skill needs no commendation. A cast was made of the heart and exudate together. The exudate was then removed from the heart while the latter was still thoroughly frozen, and a cast and model made of the heart itself. The model of the heart was then placed in the mold first made, and the intervening space filled with a plastic mixture, so that an exact model of the exudate was made. In this manner we finally obtained in each case an exact reproduction of the heart, a similar exact reproduction of the exudate, and lastly, a reproduction of the two together. A very important point relates to the size of the exudate. Most authors have contented themselves with injecting fluids, measuring only the amount as it went in, and assuming that it all remained in the pericardial sac. We did this in the beginning, but found the method grossly inaccurate, since as the injections became larger and the intrapericardial pressure higher, the pericardium would be stripped off from the great vessels posteriorly and large amounts of it would escape into the mediastinal and pleural cavities. We lost a large number of experiments in this way, and even in perfectly fresh cadavers, we have not succeeded in introducing larger amounts than 655 c.c. without producing a rupture of the pericardium, or what is more commonly the case, stripping it off the great vessels posteriorly. The exudates were measured entirely by the method of displacement, which gives, of course, absolutely accurate results.

The casts were then photographed in the studio of a commercial photographer with a large camera which was arranged vertically. The casts were placed in their proper position on the floor of the platform, using a long focus lens so that the distance was sufficiently great to prevent appreciable distortion. All of the casts were photographed at one sitting, the camera left set up, the casts were than sawn through and the cut sections brought back and photographed under precisely the same conditions. 

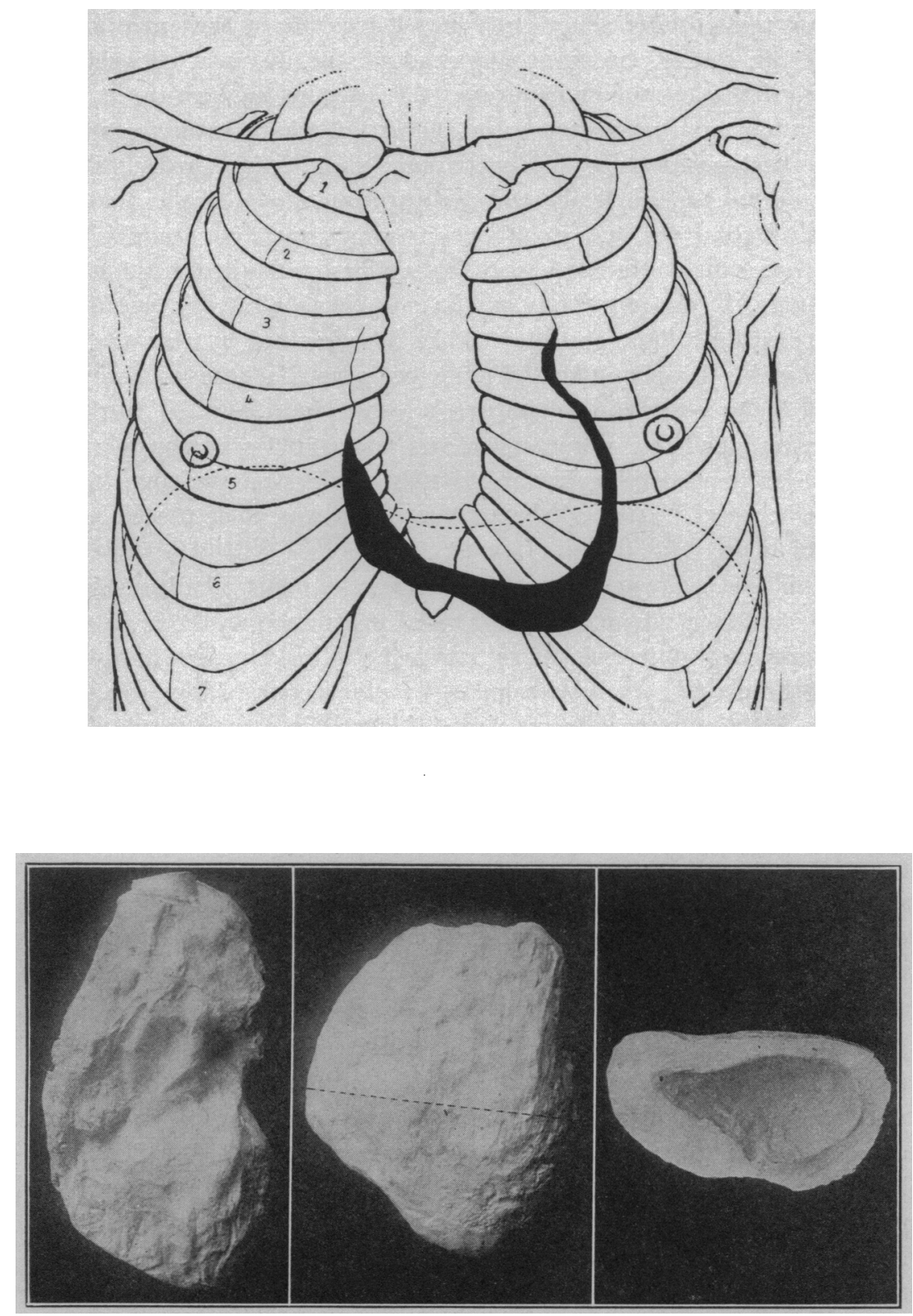

Fig. 5.-Exp. 32; 300 c.c. injected. This shows the greater part of the accumulation of fluid around the apex and diaphragmatic surface. (See note on page 206.) 
In order to be able to show accurately the outlines of the hearts and exudates on a chest outline of exactly the same relative size, the following method was adopted: First, I determined the exact amount of reduction of the photograph by comparing the size of the cast with the size of the corresponding print. From the measurements taken of the cadavers an average was struck, and all that it was necessary to do was to obtain a chest outline bearing exactly the same proportion to this average measurement as the photographs of the casts did to the casts themselves. A chest outline was then made to this exact scale. By means of tracing paper, the outlines of the heart and exudate were obtained from the photographs and these placed in position on the chest outline. The accuracy of this was insured by always leaving a considerable length of the great vessels at the base so that there could be no question as to the precise position. In addition to this, the data obtained by the measurements taken at the necropsy were at hand. For invaluable assistance in this work I am indebted to $\mathrm{Mr}$. Thomas Jones, the artist of the anatomic department of the University of Illinois.

In this way thirty-three cases were studied, and I believe that the method possesses advantages over previously used methods in that a great degree of objectivity was attained. The casts are substantial and can be examined and measured in every conceivable way. Particularly, do they show the relations of depth in a way impossible by means of the roentgen ray. My experiences lead me to believe that far reaching conclusions drawn from percussion findings, after the injection of varying amounts of fluid, are apt to be highly fallacious. It is self-evident that percussion cannot possibly give any idea of depth, and affords but a very imperfect idea, if indeed any, of fluid which accumulates between the heart and diaphragm, or in any position where the flatness of the fluid is not in contrast with the resonance of the lung.

Results Obtained.-The cases have been divided quite arbitrarily into two groups. The first group includes all those cases in which the exudate measured 350 c.c. or less; the second group included those cases in which the exudate measured between 350 and 655 c.c., the latter being the largest injection. In spite of a large number of injections with larger amounts of fluid, it was invariably found that there had been a small rupture of the pericardial sac, and that much of the fluid had escaped. This is in accordance with the experience of most authors who have made carefully controlled injections.

These two groups will be referred to as the small exudates and the medium sized exudates, respectively. Turning to the first of these, the small exudates (Experiments 4, 18, 26, 29, 32; Figs. 1, 2, 3, 4 and 

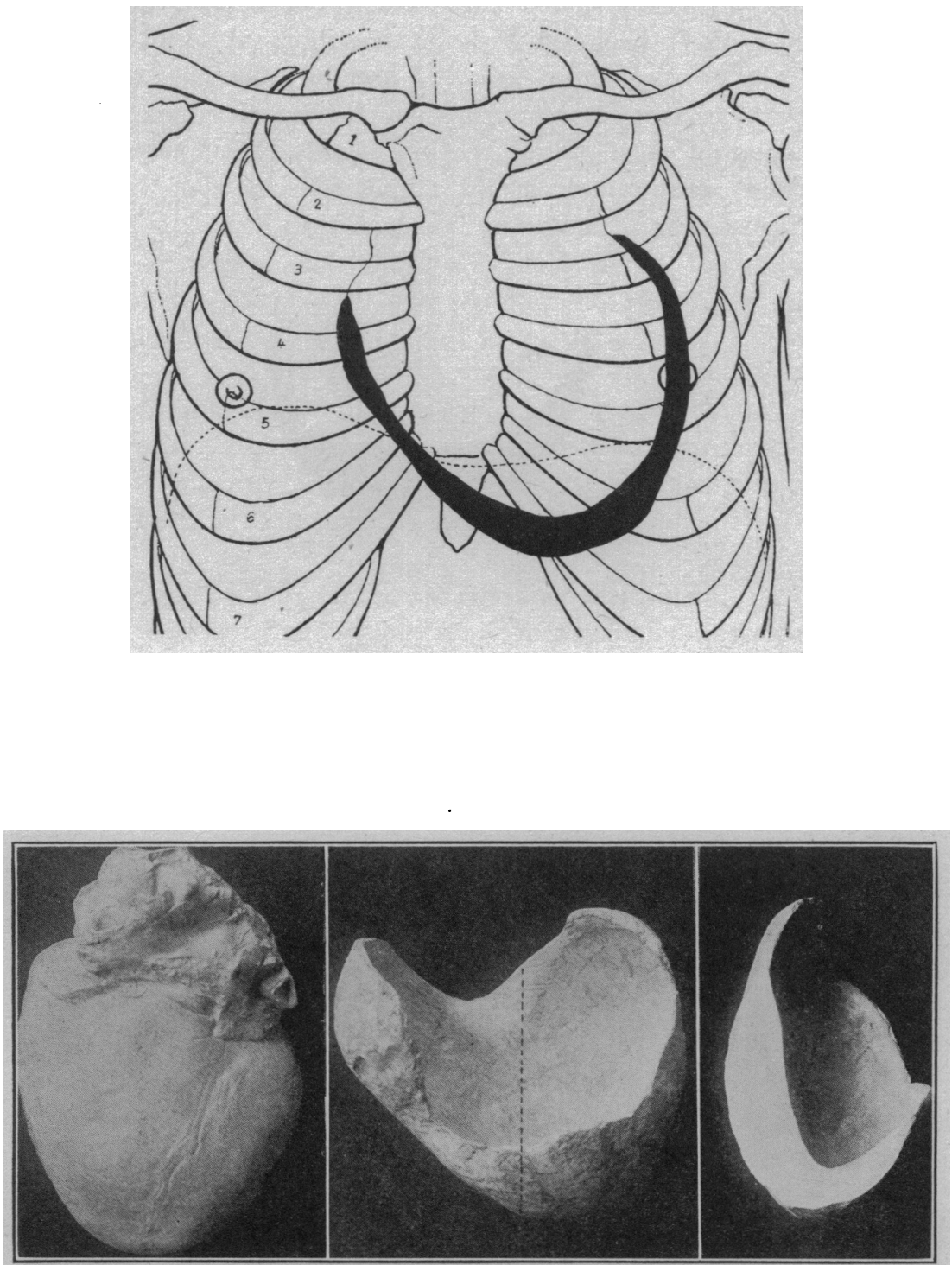

Fig. 6.-Exp. 1; 460 c.c. injected. Weight of heart $530 \mathrm{gm}$. The fluid is almost entirely confined to the diaphragmatic surface. The front of the heart is uncovered. This figure illustrates well the point that with large hearts the fluid does not collect in front. (See note on page 206.) 

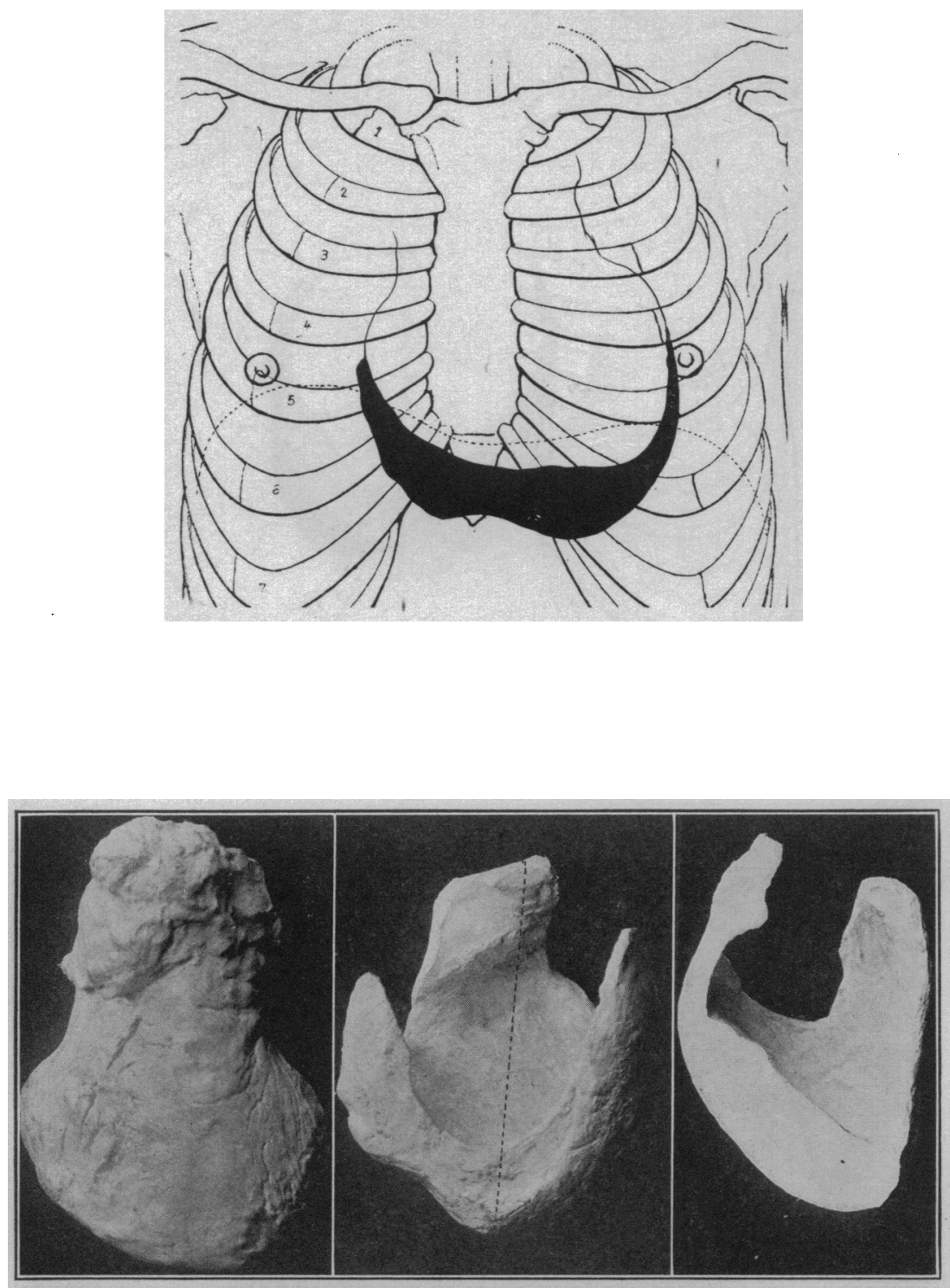

Fig. 7.-Exp. 12; 475 c.c. injected. The heart was very large, weight 550 gm. In spite of the large exudate, the entire anterior surface of the heart remained uncovered. (See note on page 206.) 

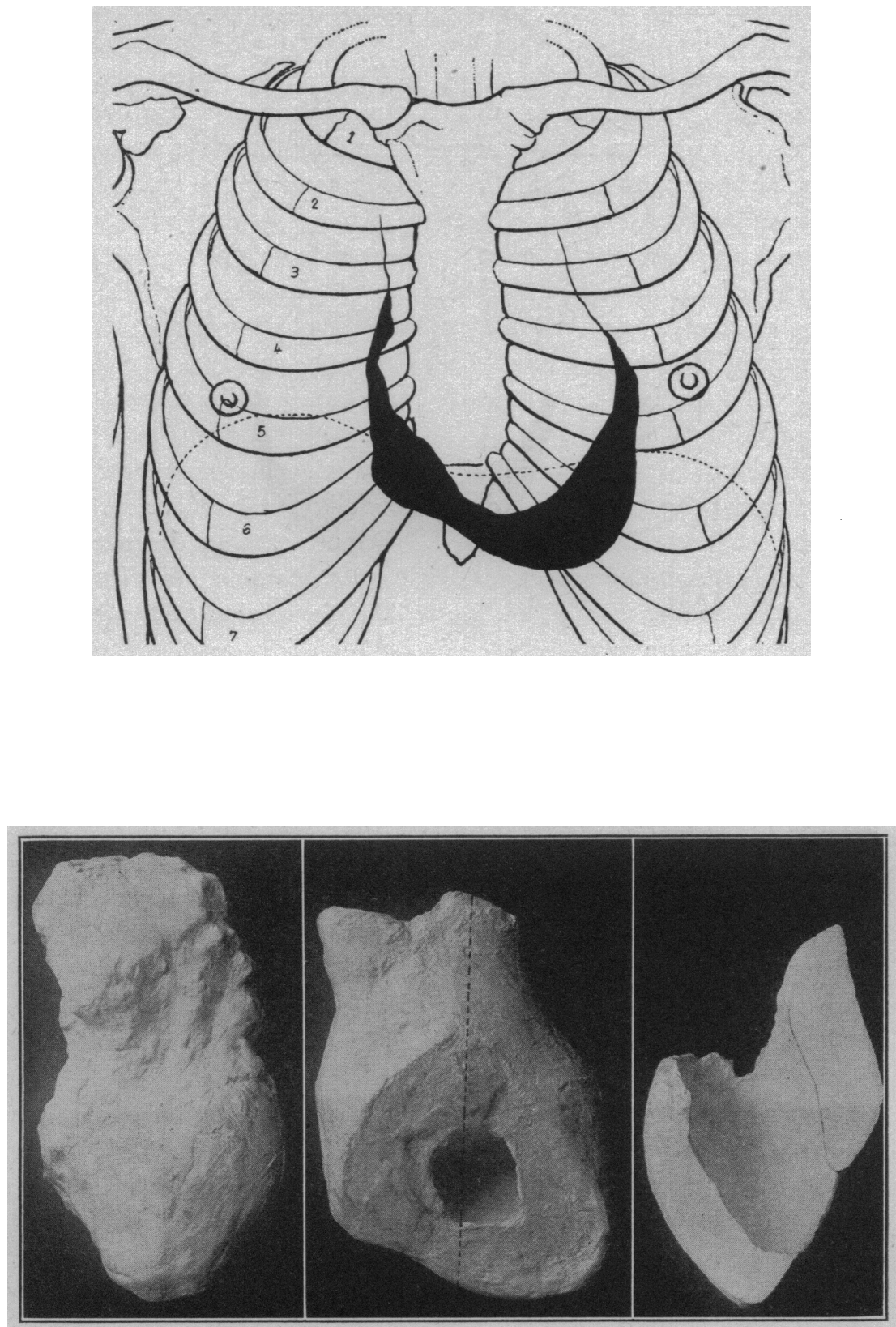

Fig. 8.-Exp. 16; 425 c.c. injected. The front of the heart is not covered by fluid, which is found in about equal amounts on the diaphragmatic surface and in front of the great vessels. (See note on page 206.) 
5 ), let us see where the exudate first collected. It is impossible, for lack of space, to reproduce all the photographs. The ones shown are selected as being typical. It requires but very slight study of the photographs and their cross sections to see that in every instance the major portion of the exudate collected in the angle formed by the anterior chest wall and the heart, and to even a larger degree between the right ventricle and the diaphragm, pushing down the latter. In every instance the apex was covered with at least as great a thickness of fluid as the remainder of the diaphragmatic surface. It should be noted that the position of the cadaver makes but little, if any, difference, since in experiments 26, 29 and 32 (Figs. 3, 4 and 5) the cadavers were injected in the upright position; the others were injected in the normal recumbent position.

A careful inspection will show that the liver was materially depressed in practically every case, especially the left lobe, as it presents in the chrondroxiphoid angle. On performing the necropsies the most striking thing noted was this pushing down of the diaphragm, and with it the left lobe of the liver. This depression of the left lobe of the liver is more in the nature of a rotation downward than a pushing down of the liver en masse.

A careful inspection of the angle formed by the pericardial exudate and the liver, shows that in not a single case was this angle obtuse, nor were we able in a single case to demonstrate to our satisfaction a sufficiently marked dullness in the fifth right interspace to be of diagnostic ialue. On the other hand, in spite of the fact that in several of the cases the exudates covered the great vessels with a fairly thick layer (as in Experiments 4 and 16; Figs. 1 and 8) we were not always able to determine this with certainty before making the postmortem. The depth at which the great vessels lie, and the readiness with which the exudate in this position may be covered by the lungs, is probably the explanation.

Turning now to the group of medium-sized exudates (Experiments $1,12,16,19,25,27$ and 28 ; Figs. 6 to 12 ) the heart is found relatively more evenly surrounded, and as the exudates grow larger in amount, the anterior surface becomes more frequently covered. In this connection it is necessary to call attention to the fact that in all work on the cadaver, inasmuch as there is no pressure due to the blood in the heart and great vessels, these structures collapse under a much smaller pressure than they would in the living subject, and in so doing become flattened out laterally and, possibly to a very slight extent, longitudinally. This is quite plainly shown in a number of the cuts, giving the appearance of the heart being relatively too large. Intravitam, the 

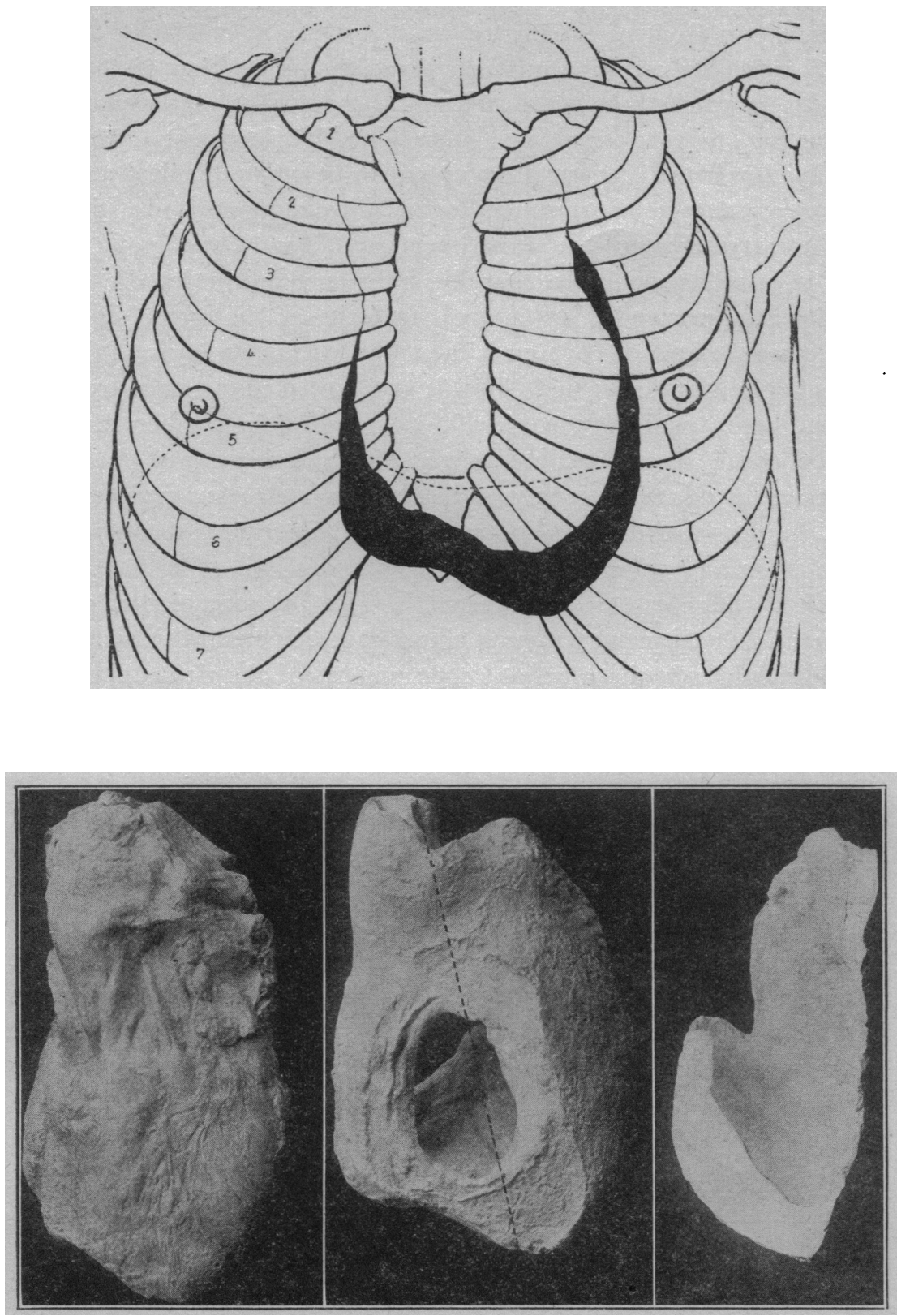

Fig. 9.-Exp. 19; 435 c.c. injected. The fuluid is largely confined to the diaphragmatic area. Note that a large part of the front of the heart is not covered by fluid. Only a small anount of fluid covers the great vessels. (See note, page 206.) 
heart would collapse much less readily, and fluid would less readily accumulate in front of the heart.

An especially careful study was made of the position of the apex beat and we reached the conclusion that there is no change whatever in its position, except, possibly, a very slight displacement downward. This is, however, of too small an extent to be of any clinical moment. In some of the cases the pushing down of the diaphragm and with it the liver, is extremely marked (Experiments 16, 25 and 28; Figs. 8, 10 and 12). It is well to emphasize that this is the outstanding feature, as disclosed by the necropsies in the great majority of the cases. In all of these cases the apex is covered with at least as thick a layer of fluid as is the diaphragmatic surface. In this group of medium sized exudates one may repeat word for word what was said of the smaller exudates, namely, that in not a single case was the cardiohepatic angle obtuse, nor were we able in a single case to demonstrate to our satisfaction a sufficiently marked dullness in the fifth right interspace to be of diagnostic value.

One of these cases, Experiment 27 (Fig. 11), occupies an unique position, in that it came from an actual case of pericarditis with effusion, developing in the course of a pneumonia of the right upper lobe. The patient died suddenly and rather unexpectedly, and within a few minutes after death we aspirated through the diaphragm in the usual way and withdrew all the fluid we could obtain, namely, 270 c.c., and inmediately injected exactly the same amount of agar solution. It may be noted that the total amount of the exudate as determined by the measurement of the cast was 405 c.c., indicating the difficulty of removing all the fluid. This case is an extraordinarily instructive one, in that the pressure conditions were precisely the same as during life. An inspection of the great veins and auricles especially shows them to be flattened out, and this must have been their condition in the last moments of life. In the light of the work of François Franck ${ }^{9}$ and Starling, ${ }^{10}$ one cannot help but believe that death in this case was actually the result of excessive pressure, and that had the fluid been aspirated, the pressure factor at least would have been removed. In this particular case a thin layer of fluid covered the heart in front, and extended far up onto the great vessels. I examined the patient about one half hour beforedeath and dictated the following note on the heart: "Apex indistinct; dullness extends outside the midclavicular line in the fifth interspace, and upward along the great vessels on the left edge

9. Franck, François: Recherches, etc.. Gaz. hebd. de méd. 29: 1877.

10. Starling: Some Points in the Pathology of Heart Disease, Lancet $1: 569,1897$. 

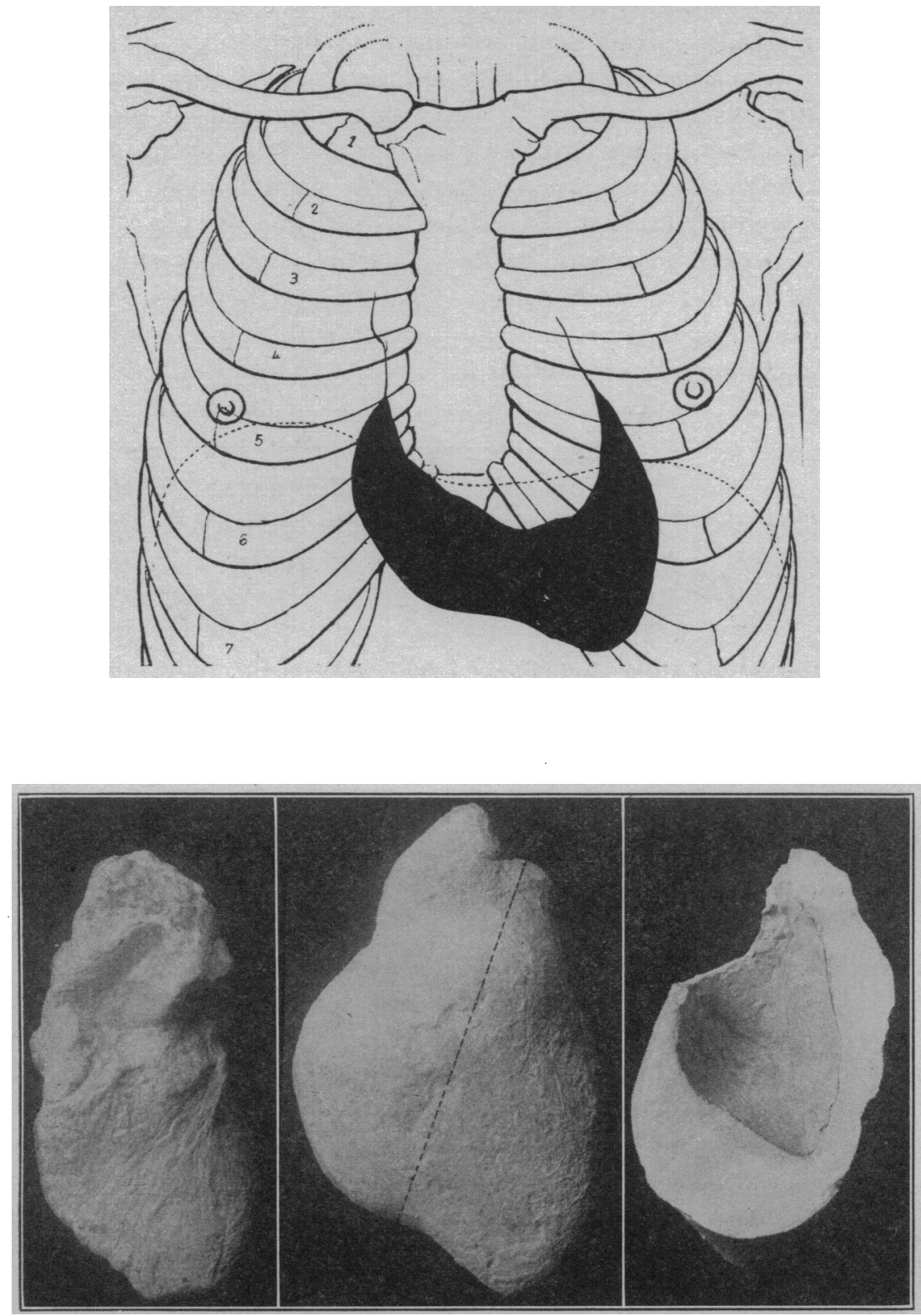

Fig. 10.-Exp. $25 ; 605$ c.c. injected. The striking feature of this experiment is the great displacement of the diaphragm downward. A moderately thick layer of fluid covers the front of the heart and the great vessels. (See note, page 206.) 
of the sternum. Right border obscured by the lung dullness. Loud to and fro friction rub over the anterior surface of the heart. Liver dullness extends two inches below the costal arch." It should be noted that this position of the liver was about an inch below what it had been on admission. A study of the photographs and section showed the heart to be surrounded with a fairly uniform layer of fluid, which, however, is somewhat thicker along the left border of the heart, and extends well up over the great vessels at the base. This fact we had been able to make out clinically. Because of the consolidation in the right lung, we could not determine with accuracy what the condition was at the cardiohepatic angle, but an inspection of the section shows that the amount of exudate there was much too small to be determined by percussion, and that the cardiohepatic angle was certainly not obtuse. I wish to lay especial stress on the pushing down of the liver, since it was on this sign, and on the retrosternal dullness that I based my diagnosis of an effusion. It should be noted especially that despite a layer of fluid, perhaps one quarter of an inch thick in front of the heart, a pericardial friction rub over this entire area could be heard plainly.

It may be said then, that the principal difference between small and medium sized exudates is that in the latter, in addition to the pushing down of the liver, the heart is more evenly surrounded by fluid. It is especially to be noted that the great vessels at the base are covered with a rather thick layer of fluid in the larger effusions of this group. This can be seen on almost any of the sections cut longitudinally. In most of the cases in which the great vessels were covered by a thick layer of fluid, this could be made out by percussion, but not in all of them, so that while this percussion dullness is to be regarded as a valuable sign when present, it cannot always be made out.

From the standpoint of the persistence of the pericardial rub, it is desirable to note in just what cases the heart remained in actual contact with the chest wall. This was the case in fourteen of the thirtythree cases. In these fourteen cases, exudates of less than 400 c.c. were found in seven, and exudates larger than this were found in the remaining seven. In these fourteen cases, therefore, it is perfectly certain that a pericardial friction rub would have been heard despite the fluid, and recalling the clinical case above enumerated, where a loud friction rub was heard over the entire heart a few minutes before death, although the necropsy disclosed a quarter of an inch of fluid between the heart and the chest wall, it is highly probable that a much larger number would have shown a friction rub despite a fair sized effusion. 

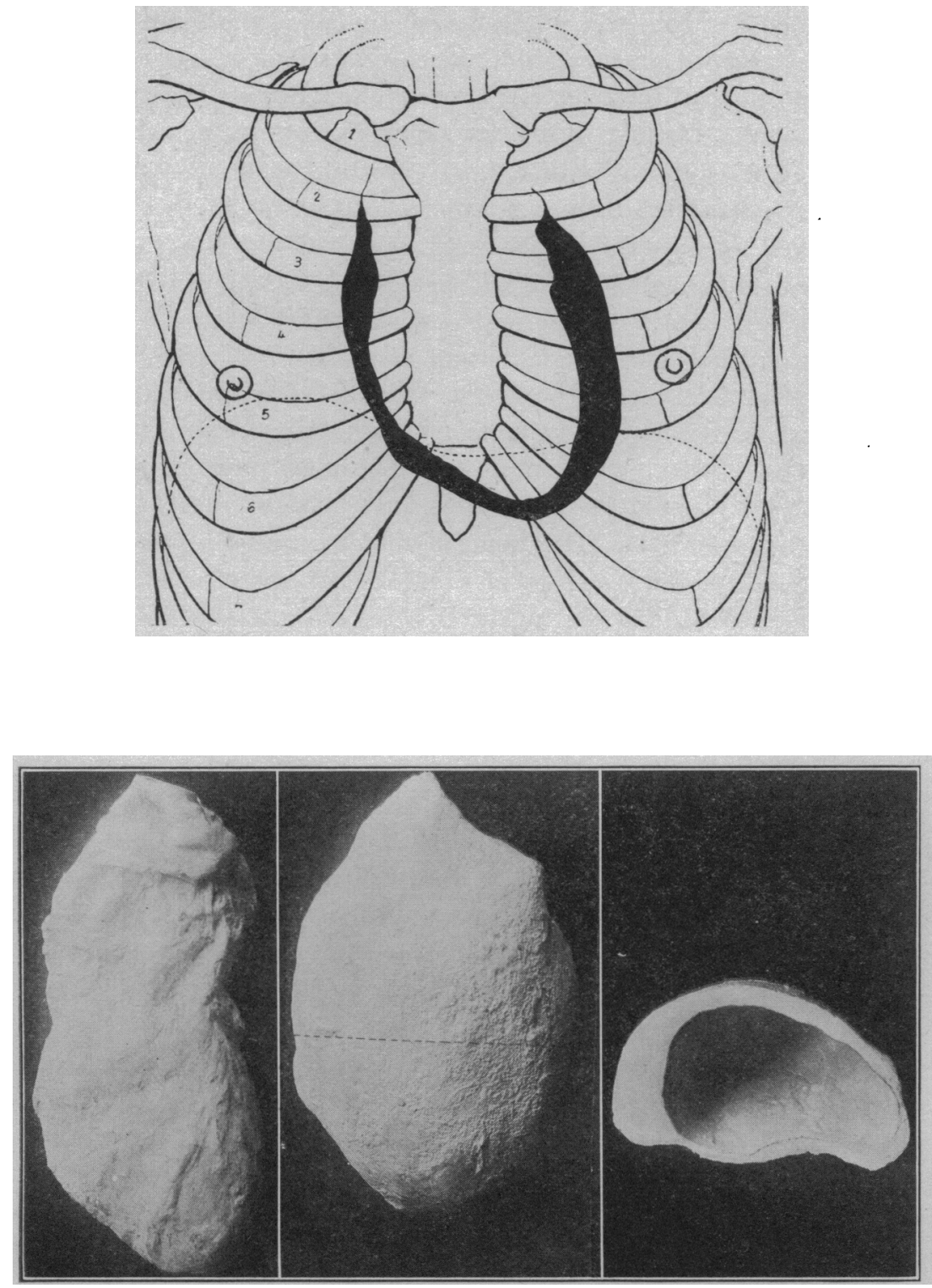

Fig. 11.-Exp. 27; 405 c.c. injected. This was an actual case of pericarditis. The fluid was drawn off immediately after death and replaced by the same amount of agar solution. In this case the exudate surrounds the heart quite uniformly, a little more along the left border. The great vessels are covered by a fairly thick layer of fluid. (See note, page 206.) 
Relation of Size of Heart and Depth of Thorax to Shape of Effusion.-It would not be profitable to give all of the numerous measurements taken as to depth of thorax, circumference of thorax, size and weight of heart, etc. Suffice it to say that I have not been able to make out any simple relationship, capable of being expressed in figures, as to the factors which cause the exudate to spare the front of the heart. Despite my inability to express this numerically, a careful inspection of the cadavers at necropsy showed that whenever the size of the heart was sufficient to fill out very fully the anteroposterior diameter of the thorax, there would be no accumulation in front of the heart. Experiments 1 and 19 (Figs. 6 and 9) illustrate this point, Experiment 12 (Fig. 7) is a striking example of it. In this individual the heart was very large, weighing $550 \mathrm{gm}$., and yet the entire front of the heart was bare of exudate, although this contained the largest exudate of the fourteen mentioned above, namely, 475 c.c. One may generalize, I believe, with safety, to this extent, and say that when the heart is relatively large, as in valvular lesions with dilatation and hypertrophy, or in cases of chronic nephritis, any exudate which may develop is likely to leave the front of the heart bare, even when the amount of fluid is quite considerable.

\section{CONCLUSIONS}

1. In pericardial effusion the fluid accumulates first along the lower margin of the heart and about the apex, particularly on the diaphragmatic surface of the heart. With small effusions, this is the only place in which fluid accumulates with regularity.

2. The result of the accumulation of the fluid in this position is to push down the left lobe of the liver. This was demonstrable in practically every case, and in many cases it was a very conspicuous feature. Special stress should be laid on this as an early diagnostic sign.

3. The second place in which fluid accumulates is over the great vessels at the base. With small effusions it is occasionally present in sufficient amount to be detected by percussion. With medium sized effusions this layer is generally thick enough to be demonstrable by percussion, and this retrosternal dullness is an important diagnostic sign.

4. With both small and medium sized exudates we were neither able satisfactorily to demonstrate percussion dullness in the fifth right interspace (Rotch), nor could a rounding of the cardiohepatic (Ebstein) angle be made out in a single case.

5. The behavior of the fluid is practically independent of the position of the patient, with effusions of the size represented by the injections. 

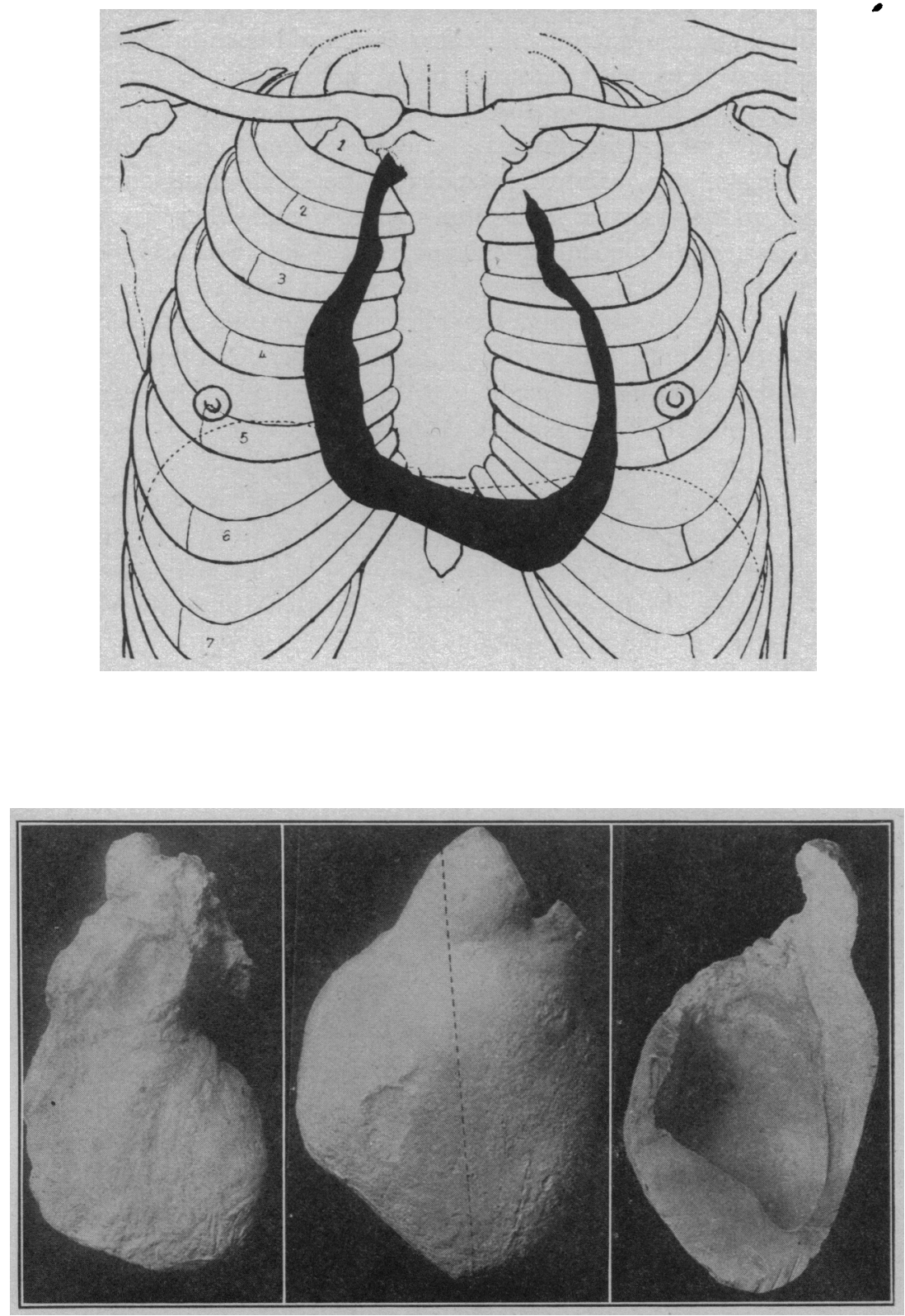

Fig. 12.-Exp. 28; 655 c.c. injected. The larger portion of the fluid is on the diaphragmatic surface and over the great ressels. (See note, page 206.) 
6. In at least fourteen of the thirty-three cases the anterior surface of the heart, in spite of the exudate, remained, in part, uncovered by the fluid, so that a pericardial friction rub could readily exist. This persistence of the pericardial rub is to be anticipated in cases in which the heart is relatively large, so that it fills out the space between the vertebral column and the sternum.

7. From the standpoint of most readily reaching small amounts of fluid, the most appropriate sites for puncture are either just outside the apex or in the chondroxyphoid angle. 